\title{
Neunter Beitrag zur Coleopteren-Fauna der Philippinen.
}

\section{Von W. Schultze, Manila.}

(Mit Tafel 1.)

Im folgenden gebe ich die Beschreibungen einer Anzahl neuer Arten, die zum gröfsten Teil aus der Zentral-Provinz Bukidnon der Insel Mindanao stammen. Unter Berücksichtigung und Durchsicht des reichhaltigen in Frage kommenden CurculionidenMaterials des Dresdener Zoologischen Museums gelang es, eine Anzahl von zweifelhaften Arten richtigzustellen, wobei Prof. K. M. Heller mich in freundlichster Weise unterstützte und wofür ich ihm auch an dieser Stelle danken möchte.

\section{Cerambycidue.}

Prionomma mindanaona sp. nov. Taf. 1, Fig. 6.

Kopf und Halsschild schwarz, Flügeldecken dunkel rotbraun. Kopf sehr dicht, grob und unregelmälsig punktiert, die Stirn grubig vertieft, die Fühleransätze kräftig skulptiert. Die feine Mediallängsfurche reicht bis zum Scheitel. Fühler in Länge bis zum apicalen Drittel der Decken reichend. Erstes Glied glänzend, sparsam grob narbig punktiert, zweites bis sechstes Fühlerglied oberseits glänzend, mit vereinzelter Punktierung, seitlich matt lederartig. Die folgenden Glieder oberseits mit narbigen Längseindrücken. Das dritte Glied am längsten, ungefähr ein Drittel länger wie das erste oder vierte Glied. Halsschild lederartig gerunzelt, im diskalen Teil vor der Mitte mit zwei rundlichen Schwellungen und je seitlich einer weiteren, die bis nahe an den Hinterrand reicht. Seitenrand mit drei Dornen bewehrt, zwei davon vor der Mitte, der mittlere Dorn am längsten, der kürzeste an den Hinterecken gelegene ist etwas nach hinten gerichtet. Schildchen dicht narbig punktiert. Die Flügeldecken fein lederartig gerunzelt und weitläufig punktiert. Vorder-, Mittel- und Hinterbrust dicht hell bräunlich behaart. Abdominalsegmente glänzend und fein weitläufig punktiert. Letztes Segment die Decken weit überragend.

․ Länge : $44 \mathrm{~mm}$. Schulterbreite : $14,2 \mathrm{~mm}$.

M ind a n a o, Prov. Bukidnon, Lindaban (M. Ramos).

Typus in meiner Sammlung.

Euclea pulchella sp. nov. Taf. 1, Fig. 7.

Kopf, Halsschild und Beine, mit Ausnahme der Tarsen, dunkel rötlich kupferbronzefarbig, sehr glänzend, die Flügeldecken mit grünem Schiller. Die Tomentbänder und Flecken creme-weifs. 
Kopf mit einer undeutlichen Längsschwiele, zerstreut punktiert. Zwei Längsbänder laufen divergierend bis zum Scheitel und bilden die Figur V. Erstes Glied der Fühler kräftig verworren punktiert, die vordere Hälfte des vierten Gliedes creme-weifs, die folgenden Glieder schwarz pubesziert. Halsschild kräftig zerstreut punktiert, nächst des Vorderrandes ein schmales Tomentband und ein weiteres nächst dem Hinterrande, letzteres in der Mitte unterbrochen. Flügeldecken gleichmäfsig zerstreut punktiert. Hinter dem Schildchen ein ungefähr dreieckiger Nahtfleck. In der Mitte zwei breite Querbänder, die an den Seitenrändern zusammenlaufen. Am Beginn des apicalen Drittels ein schmäleres Querband, welches zusammenfliefsend mit einem Streifen längs der Naht und einem Streifen längs des Aufsenrandes das Spitzendreieck umschreiben. Im Spitzendreieck ein kurzer Längsstreifen. Die Schultern werden durch einen weiteren, vom Basalrand bis zum Seitenrand reichenden schmalen Streifen umschrieben. Tarsen dunkelblau.

Länge : $16 \mathrm{~mm}$. Breite : $6 \mathrm{~mm}$.

Mindana o, Prov. Bukidnon, Lindaban (W. Schultze).

Typus in meiner Sammlung.

Diese Art unterscheidet sich durch ihre sonderbare Färbung von den anderen philippinischen Euclea-Arten und erinnert aus diesem Grunde stark an Arten der Gattung Aprophata.

\section{Curculionidae.}

Pachyrrhynchus taylori sp. nov. Taf. 1, Fig. 1 ㅇ, 2 or.

Mit $P$.gemmatus Waterh. verwandt, jedoch gröfser und relativ schlanker gebaut. Stark glänzend, tief glutrot mit violetten Reflexen und grofsen Augenmakeln auf dem Halsschild und den Flügeldecken. Rüssel im apicalen Teil breiter als an der Basis, die basale Hälfte mit einem flachen Eindruck, einen grofsen goldigen Schuppenfleck tragend. Stirn mit einem runden Schuppenfleck. Eine weitere Makel an der Seite des Rüssels und unterhalb des Auges. Halsschild etwas länger wie breit, die gröfste Breite etwas vor der Mitte. Am Hinterrand in der Mitte eine Schuppenmakel, je seitlich nächst dem Vorderrand eine gröfsere Makel und an den Seiten eine sehr grofse Makel, die vom Vorderbis zum Hinterrand reicht. Flügeldecken beim $q$ breit, gedrungen oval, beim $\sigma^{7}$ länglich, gestreckt oval, mit 16 grofsen Schuppenmakeln, d. h. jede Decke mit 7 und 2 gemeinsame auf der Naht. Zwei Makeln im basalen Teil, zwei in der Mitte, eine weitere etwas langgestreckte am Seitenrand, zwei weitere im apicalen Teil und zwei Makeln, ebenfalls in der hinteren Hälfte, auf der 
Naht. Beim $q$ sind die Schuppen im äufseren Teil jeder Makel rotgoldig, im inneren Teil heller und dunkler blau und grün, edelsteinartig glitzernd. Beim $\sigma^{\top}$ sind die Makeln so vergröfsert, dafs sie aneinander stofsen, und die Beschuppung ist eine rötlich goldige. Mittel- und Hinterbrust, sowie erstes Abdominalsegment je seitlich mit einem Schuppenfleck. Schenkel mit einem Schuppenfleck nächst den Kniegelenken.

$\sigma^{7}$. Länge : $17,5 \mathrm{~mm}$, Breite: $6,5 \mathrm{~mm}$. ㅇ. Länge: $18 \mathrm{~mm}$, Breite : $8 \mathrm{~mm}$.

L u z o n, Prov. Kalinga, Balbalin (Edw. Taylor).

Typen in meiner Sammlung.

Diese mir bekannte farbenprächtigste Pachyrrhynchus-Art widme ich ihrem Entdecker, dem Reptilienforscher der Philippinen, Herrn Edward Taylor, welcher diese Art zusammen mit dem an anderer Stelle beschriebenen Macrocyrtus kalinganus sp. $\mathrm{n}$. fand und wegen der Farben- und Zeichnungsähnlichkeit für einẹ Art hielt.

Pachyrrhynchus amabilis sp. nov. Taf. 1, Fig. 10.

Kopf, Halsschild und Beine mit Ausnahme der Tarsen stark glänzend glutrot. Flügeldecken matt dunkelgrün. Die Querbänder des Halsschildes, sowie die sehr grofsen Flecken auf den Flügeldecken hell rosafarbig.

Rüssel länger als breit, in der Mitte stufig abgesetzt, mit einem Längseindruck, welcher von der Mitte an in der basalen Hälfte grubig erweitert ist und einen länglichen Schuppenfleck trägt. Der Rüssel im apicalen Teil kräftig und unregelmäfsig, nach der Stirn zu schwächer und verlaufend punktiert. Fühler dunkelgrün. Ein kleiner Schuppenfleck unterhalb des Auges. Halsschild so lang wie breit, mit einem Vorderrandband und einem in der Mitte erweiterten Band nächst dem Hinterrande. Beide Bänder an den Seiten fleckenartig zusammenlaufend. Decken dunkel bläulichgrün, nicht glänzend, mit deutlichen Punktreihen. Jede Decke mit einem grofsen Schuppenfleck auf der vorderen und hinteren Hälfte. Diese Flecken nehmen den gröIsten Teil der Decke ein und reichen seitlich von der ersten Punktreihe nächst der Naht bis zum Aufsenrand. Die nicht befleckten Teile der Flügeldecken bilden eine kreuzförmige Zeichnung. Unterseite mit einem Schuppenfleck je seitlich auf der Mittel- und Hinterbrust. Beine unregelmäfig punktiert, die Tarsen dunkel bläulichgrün.

Länge: $14,5 \mathrm{~mm}$. Breite: $6 \mathrm{~mm}$.

M i n d a n a o, Prov. Bukidnon, Lindaban (W. Schultze).

Typus in meiner Sammlung. 
Diese Art ist von allen bekannten Pachyrrhynchus-Arten leicht durch ihre besonders auffällige Färbung und Zeichnung zu unterscheiden.

Pachyrrhynchus chamissoi sp. nov. Taf. 1, Fig. 9, ㅇ.

Kopf, Halsschild und Beine glänzend, dunkel glutrot mit violettem Schimmer. Flügeldecken matt fettglanzartig bläulich schwarz. Rüssel breiter am Apex als an der Basis, in der Mitte stufig abgesetzt, Basalhälfte mit einer grubigen Vertiefung, die Seitenränder kräftig wulstig hervortretend. Die Vertiefung mit einem blafs grünlich weifsen Schuppenfleck. Halschild so breit wie lang, fein zerstreut punktiert, mit einer undeutlichen Furche nächst dem Vorderrand und einer deutlichen nächst dem Hinterrand. An den Seiten je zwei Flecke. Flügeldecken mit je neun Punktreihen. Vor der Mitte ein grofser querbandartiger, blafs grünlich weifser Schuppenfleck, seitlich vom zweiten bis zum achten Spatium reichend, o. Der Fleck wird von den Punktreihen durchbrochen. Im Apicaldreieck ein länglicher und zwei rundliche Flecke. Mittel- und Hinterbrust mit je einem Schuppenfleck seitlich. Tarsen bläulich schwarz. $\sigma^{7}$ ohne jede Fleckenzeichnung auf den Flügeldecken.

q. Länge : $13,5 \mathrm{~mm}$. Breite : $6 \mathrm{~mm}$. $\sigma^{7}$. Länge : $12 \mathrm{~mm}$. Breite : $5 \mathrm{~mm}$.

M i nda na o, Prov. Bukidnon, Lindaban (W. Schultze).

Typen in meiner Sammlung.

Diese Art variiert ziemlich stark (q), der grofse querbandartige Fleck ist teilweise aufgelöst in eine Reihe von kleineren Flecken, drei bis vier in Anzahl. Auch sind die Flecke im Apicaldreieck teilweise oder nicht vorhanden.

Diese Art widme ich dem Andenken an Adelbert von Chamisso, Dichter von: „Die alte Waschfrau“, welcher vor über hundert Jahren (17. Dezember 1817, Manila Bai) die Philippinen als Mitglied der Romanzoff-Expedition mit der Brigg Rurik in Gesellschaft des Entomologen Eschscholtz besuchte.

$$
\text { Pachyrrhynchus apocyrtoides sp. nov. }
$$

Verwandt mit $P$. erichsoni Waterh. Kopf bläulich schwarz, Halsschild und Flügeldecken dunkelbraun mit violettem Schiller. Rüssel in der Mitte querstufig abgesetzt, in der Basalhälfte mit einer kurzen, kräftig ausgeprägten Längsfurche, die kaum bis zur Stirn reicht. Halsschild glänzend, etwas breiter wie lang, die Seiten gleichmäfsig gerundet. Ein schmaler cremefarbiger Schuppenstreifen am Vorderrand verläuft in eine Makel an den Seiten. Je seitlich der Mitte auf der Scheibe ein kleiner runder 
Schuppenfleck. Flügeldecken matt, fein lederartig gerunzelt mit gut ausgeprägten Längspunktreihen. Jede Decke mit drei Gruppen von makelartigen Schuppenschwärmen, deren Ränder nicht scharf markiert sind, zwei befinden sich nächst der Basis, eine grofse unregelmälsig querbindenartige Makel in der Mitte und fünf im apicalen Teil.

${ }^{7}$. Länge : $11 \mathrm{~mm}$. Breite : $4 \mathrm{~mm}$.

M ind a n a o, Prov. Bukidnon, Lindaban (W. Schultze).

Typus in meiner Sammlung.

Pachyrrhynchus bucasanus sp. nov. Taf. 1, Fig. 11.

Aus der Verwandtschaft von $P$. erichsoni Waterh., glänzend dunkel weinrot mit violetten Reflexen und grofsen blafs gelblichgrünen Schuppenmakeln. Rüssel dem von $P$. apocyrtoides $\mathrm{m}$. sehr ähnlich. Halsschild so lang wie breit, mit einem Vorderrandschuppenstreifen und einem kurzen Streifen oberseits am Hinterrand. Je seitlich in der Mitte eine grofse, ungefähr dreieckige Schuppenmakel, an den Seiten eine grofse runde Makel. Flügeldecken kurz und gedrungen, kräftig gewölbt, mit schwach ausgeprägten Punktreihen. Jede Decke mit neun Schuppenmakeln, zwei an der Basis, zwei in der Mitte, vier weitere bilden eine Querreihe am Beginn des apicalen Drittels und eine dreieckige Makel im Spitzendreieck. Beine kupferig glänzend.

+. Länge : $13 \mathrm{~mm}$. Breite: $6,5 \mathrm{~mm}$.

Insel Bucas (W. Schultze).

Typus in meiner Sammlung.

Diese Art gehört zu den gedrungensten Formen dieser Gattung.

Pachyrrhynchus atrocyaneus sp. nov. Taf. 1, Fig. 12, ‥

Glänzend dunkelblau, mit cremefarbigen Schuppenmakeln. Rüssel in der Basalhälfte mit einer stark ausgeprägten, ungefähr quadratischen Grube. Halsschild länger wie breit, die grölste Breite vor der Mitte, mit einer kräftigen Furche nächst dem Hinterrand. Ein schmaler Schuppenstreifen längs des Vorderrandes fortgesetzt an den Seiten bis zum Hinterrand reichend. In der Mitte an der Hinterrandfurche ein dreieckiger Schuppenfleck. Flügeldecken herzförmig nach dem Apex zu stark verjüngt, mit sehr kräftigen furchenartigen Längspunktreihen, so dafs die Zwischenräume erhaben hervortreten. Jede Decke mit neun Schuppenflecken und einer Längsmakel am Aufsenrande. Fünf Flecken befinden sich in der Basalhälfte, drei bilden eine Querreihe im letzten Drittel und ein Fleck im Spitzendreieck. Beine schwarz. 
๙7. Länge : $9 \mathrm{~mm}$. Breite : $3,8 \mathrm{~mm}$. ㅇ. Länge : $12 \mathrm{~mm}$. Breite : $5 \mathrm{~mm}$.

M i nd a n a , Prov. Zamboanga, Zamboanga (W. Schultze).

Typen in meiner Sammlung.

Diese Art bildet eine Rand- oder Übergangsform $\mathrm{zu}$ anderen nächst verwandten Gattungen.

Pachyrrhynchus signaticollis sp. nov. Taf. 1, Fig. 13.

Verwandt mit $P$. erichsoni Waterh. Kopf, Halsschild und Beine glänzend, Flügeldecken matt schwarz. Rüssel in der Mitte stufig abgesetzt, Basalhälfte mit einer langgestreckten bis zur Stirn reichenden Grube und einer sehr kräftig ausgeprägten Mittelfurche,-welche bis an den Scheitel reicht. Halsschild in der Mitte mit einer blafsrosafarbigen Schuppenquerbinde, welche auf der Scheibe unterbrochen und an den Seiten makelartig erweitert ist. Flügeldecken mit regelmäfsigen Längspunktreihen. Jede Decke mit neun runden blafs rosafarbigen Schuppenflecken, die in der schematischen Anlage wie bei $P$. bucasanus $\mathrm{m}$. gestellt sind.

Länge : $11 \mathrm{~mm}$. Breite : $4,5 \mathrm{~mm}$.

M indana o, Prov. Agusan, Butuan (C. M. Weber).

Typus in meiner Sammlung.

$$
\begin{gathered}
\text { Pachyrrhynchus ardentius }{ }^{1} \text { ) ssp. corpulentus nov. } \\
\text { Taf. 1, Fig. 14, }+ \text {. }
\end{gathered}
$$

Glänzend glutrot, Schuppenzeichnungen blafs gelblichgrün. Flügeldecken gedrungen und mehr gewölbt als in der Nominatform. Halsschild je seitlich der Mitte mit einem Längsschuppenstreifen. Die Makeln der Flügeldecken im Vergleich zur Nominatform längsstreifen- oder querbindenartig ausgezogen und zusammenfliefsend.

๙ॅ. Länge: $13 \mathrm{~mm}$. Breite : $5,6 \mathrm{~mm}$. ㅇ. Länge : $14,5 \mathrm{~mm}$. Breite : $7 \mathrm{~mm}$.

M ind a na o, Prov. Bukidnon, Lindaban (W. Schultze).

Typen in meiner Sammlung.

$$
\text { Macrocyrtus multipunctatus sp. nov. }
$$

Glänzend schwarz. Rüssel gleichbreit, relativ langgestreckt, mit einem flachen Grübchen, welches in eine bis zur Stirn reichende Längsfurche ausläuft. Stirn mit einem blafsgrünen Schuppenfleck. Halsschild etwas länger wie breit, mit einem irregulären Vorderrandstreifen, welcher auf den Seiten mit einem unregelmäfsigen Schuppenfleck zusammenläuft. Je seitlich der

1) Philippine Journ. Sci. (1919), Vol. XV, 550, Pl. I, fig. 7, + . 
Mitte am Hinterrand ein dreieckiger Schuppenfleck. Flügeldecken länglich oval, am Apex verrundet, mit einer grofsen Anzahl kleiner blafsgrüner Schuppenflecke, die im basalen Drittel unregelmälsig zerstreut angeordnet, in der Mitte unregelmälsig querbindenartig und im apicalen Drittel ebenfalls unregelmäfsig zerstreut sind. Hinterschienen an der Unterseite mit vier kräftigen stumpfen Zähnchen besetzt.

$\sigma^{7}$. Länge: $14 \mathrm{~mm}$. Breite: $6,5 \mathrm{~mm}$.

L u z o n, Prov. Nueva Viscaya, Imogen (Georg Boettcher). Typus in meiner Sammlung.

Diese Art unterscheidet sich leicht durch die sonderbare Zeichnung von den anderen Arten dieser Gattung. Ich erhielt dieselbe durch die Güte des Herrn Eduard Vofs, Spandau.

Macrocyrtus trivittatus sp. nov. Taf. 1, Fig. 4, or.

Matt schwarz, mit blafsgrünlichen Schuppenstreifen. Rüssel verworren punktiert, mit einem grofsen rundlichen Eindruck in der Basalhälfte nebst Schuppenfleck, sowie eine feine Längsfurche, welche bis zur Stirn reicht. Halsschild so lang wie an der Basis breit. Je seitlich der Mitte am Hinterrand ein makelartiger Schuppenschwarm, beim $q$ gröfser als beim $0^{7}$, an den Seiten über den Vorderhüften ein Schuppenfleck. Flügeldecken beim $\sigma^{7}$ länglich oval, am Apex verrundet, beim $q$ gedrungen oval, jede Decke in eine stumpfe Spitze auslaufend, dadurch am Apex einen kleinen dreieckigen Ausschnitt bildend. Die Oberfläche fein lederartig gerunzelt, mit undeutlichen Punktreihen. Jede Decke mit drei beim $\sigma^{7}$ schmäleren, beim $q$ breiteren Längsschuppenstreifen, die im apicalen Dreieck zusammenlaufen. Ein Streifen läuft von der Basis parallel zur Naht bis zum Apex, der zweite längs des seitlichen Deckenabsturzes und der dritte längs des Seitenrandes. Hinterschienen beim $\sigma^{\top}$ unterseits kräftig behaart.

$\sigma^{7}$. Länge: $16 \mathrm{~mm}$. Breite : $6,5 \mathrm{~mm}$. ㅇ. Länge: $16 \mathrm{~mm}$. Breite: $7 \mathrm{~mm}$.

$\sigma^{7}$ von Luzon, Prov. Benguet, Berg Pulog (W. Schultze), $q$ von Prov. Nueva Viscaya, Imogen (Georg Boettcher).

Das von Boettcher gesammelte Stück wurde mir von Herrn Eduard Vofs gütigst überlassen.

Besonders erwähnen möchte ich, dafs obige Art Pachyrrhynchus modestior Behr. und $P$. igorota $\mathrm{m}$. sehr ähnlich sieht.

Macrocyrtus kalinganus sp. nov. Taf. 1, Fig. 3, ㅇ.

Glänzend pechbraun, mit violetten Reflexen und grofsen Schuppenmakeln, dem Pachyrrhynchus taylori m. sehr ähnlich. 
Rüssel zerstreut punktiert, mit flachem, bis zur Stirn reichenden Eindruck und einer scharf ausgeprägten Längsfurche. Der Eindruck trägt eine grofse Schuppenmakel. Halsschild so lang wie breit. Je seitlich am Hinterrand ein kleiner und etwas vor der Mitte ein grofser rundlicher Schuppenfleck. Seiten mit einer sehr grofsen Schuppenmakel, vom Vorder- bis zum Hinterrand reichend. Flügeldecken beim $q$ breit, gedrungen oval, im apicalen Teil stark verschmälert, jede Decke in eine stumpfe Spitze auslaufend und am Nahtende einen dreieckigen Ausschnitt bildend; beim $\sigma^{7}$ länglich gestreckt oval, die Decken am Apex verrundet. Jede Decke mit 8 Schuppenmakeln und einer kleinen Nahtmakel im apicalen Teil, zwei an der Basis, zwei in der Mitte, eine langgestreckte am Seitenrande und drei sowie die gemeinsame Nahtmakel im apicalen Drittel jeder Decke. Beim o läuft die grofse Randmakel mit der Makel im Spitzendreieck zusammen. Die Beschuppung in der äufseren Zone jeder Makel ist rotgoldig, in der inneren blaugrün. Schenkel mit einem Schuppenfleck nächst den Kniegelenken.

$\sigma^{7}$. Länge : $16,3 \mathrm{~mm}$. Breite : $6 \mathrm{~mm}$. ․ Länge : $18 \mathrm{~mm}$. Breite : 7,5 mm.

L u z o n, Prov. Kalinga, Balbalin (Edw. Taylor).

Typen in meiner Sammlung.

Diese Art und Pachyrrhynchus taylori m. stellen wohl unter den sogenannten Mimikriformen dieser Gruppe die am stärksten ausgeprägten Vertreter dar.

Metapocyrtus (Orthocyrtus) lanusinus sp. nov.

Taf. 1, Fig. 8, ㅇ.

Mit $M$. insulanus $\mathrm{m}$. verwandt, jedoch schlanker in Form, glänzend schwarz, mit blafs grünlich-weifser Schuppenzeichnung. Die Skulptur des Rüssels ähnlich wie bei obiger Art, der Schuppenfleck bis zur Stirn reichend. Halsschild länger als breit, viel feiner und gleichmäfsiger punktiert als bei $M$. insulanus, mit einem Querband am Vorder- und Hinterrand, welche am Seitenrand zusammenlaufen. In der Mitte ein anderes Querband, unregelmäfsig fleckenartig erweitert, im diskalen Teil unterbrochen. Flügeldecken weitläufig punktiert. Jede Decke mit einer feinen Schuppenlinie längs der Naht, sowie vier Längsstreifen, die mehr oder weniger unterbrochen sind, Zwischen den primären Längsstreifen befinden sich rudimentäre sekundäre Streifen, welche im ersten und zweiten Spatium (o) zu Flecken reduziert sind und im dritten und vierten Spatium eine Reihe von länglichen Flecken bilden. Beim $\sigma^{7}$ sind alle Streifen breiter, die sekundären nur 
in der Deckenmitte als rundliche oder längliche Flecken vorhanden und bilden eine querbandartige Reihe.

$\sigma^{7}$. Länge : $14,5 \mathrm{~mm}$. Breite : $5,5 \mathrm{~mm}$. ․ Länge: $16,5 \mathrm{~mm}$. Breite : $6,5 \mathrm{~mm}$.

M ind a n a o, Prov. Bukidnon, Lindaban (W. Schultze).

Typen in meiner Sammlung.

Rhinoscapha iligana sp. nov. Taf. 1, Fig. 5.

Mit $R$. merrilli ${ }^{1}$ ) m. nahe verwandt, schwarz, mit blafs metallischgrüner, auf dem Rüssel, Prothorax, sowie den Beinen grünlich blauer Beschuppung. Rüssel kürzer und breiter an der Basis als bei $R$. merrilli, dicht punktiert, die glatte Längsschwiele stärker hervortretend als bei obiger Art, mit einer punktförmigen Einpressung auf der Stirn. Prothorax kürzer und gedrungener, die verworrene Punktierung schwächer als bei voriger Art. Die Mittellängsschwiele des Rüssels ist auf dem Halsschild fortgesetzt und stark ausgeprägt. Jede Flügeldecke mit fünf primären Längsleisten und zwischen denselben mit fünf sekundären Leisten. Die letzteren verkürzt (erste, dritte und vierte) oder rudimentär vorhanden (zweite und fünfte). Die Zwischenräume zwischen den primären Leisten gleichmälsig punktiert und mit zwei Längsreihen von groben punktförmigen Einpressungen.

ๆ. Länge : $20 \mathrm{~mm}$ mit Rüssel. Schulterbreite : $7,3 \mathrm{~mm}$.

M in dan a o, Prov. Lanao, Iligan (Dionisio Vidal).

Typus in meiner Sammlung.

$R$. iligana unterscheidet sich von $R$. merrilli besonders dadurch, dafs bei der ersteren keinerlei Querleisten zwischen den Längsleisten vorhanden sind, während bei der letzteren immer, wie ich an einer grofsen Anzahl von Exemplaren feststellen konnte. Auch sind die Gattungscharaktere von Rhinoscapha und Celebia ineinander übergehende und recht relative $\mathrm{zu}$ nennen.

\section{Bemerkungen zu einigen Arten.}

Pachyrrhynchus gemmatus Chevr. und var. ardens Chevr. sind zu $P$. orbifer Waterh. zu stellen.

Pachyrrhynchus sanchezi Heller $=P$. immarginatus Kraatz. Das als $\sigma^{7}$ von Pachyrrhynchus absurdus Schultze beschriebene Stück ist nach genauer nochmaliger Untersuchung ein $\sigma^{\top}$ von $P$. speciosus Waterh. und der wahre $P$. absurdus $\sigma^{7}$ noch nicht bekannt, beide Arten wurden von G. Boettcher, sowie von meinem Sammler an derselben Pflanze gefunden.

1) Philippine Journ. Sci. (1919), Vol. XV, 557, Taf. I, fig. 16. 
Metapocyrtus violaceus Schultze $=M$. bifasciatus Waterh.

Metapocyrtus carinatus Schultze $=M$. cylas Heller.

Eupyrgops banahaonis Heller $=E$. semperi. Faust.

Xylotrupes mindanaoensis Schultze ist in die Gattung Myrina Redtenb. zu stellen und ist nahe verwandt mit $M$. pfeifferi Redtenb. von Borneo.

\section{Erklärung zu Tafel 1.}

Fig. 1. Pachyrrhynchus taylori sp. nov. (q).

" 2. Pachyrrhynchus taylori sp. nov. $\left(\sigma^{7}\right)$.

" 3. Macrocyrtus kalinganus sp. nov. (q).

" 4. Macrocyrtus trivittatus sp. nov. ( $\left.\sigma^{7}\right)$.

” 5. Rhinoscapha iligana sp. nov. ().

"6. Prionomma mindanaona sp. nov. (q).

"7. Euclea pulchella sp. nov.

" 8. Metapocyrtus lanusinus sp. nov. (q).

" 9. Pachyrrhynchus chamissoi sp. nov. (q).

„10. Pachyrrhynchus amabilis sp. nov. (ه7).

"11. Pachyrrhynchus bucasanus sp. nov. ().

"12. Pachyrrhynchus atrocyaneus sp. nov. (q).

"13. Pachyrrhynchus signaticollis sp. nov.

"14. Pachyrrhynchus ardentius ssp. corpulentus nov. (q).

(Sämtliche Figuren aufser Nr. 6 in $1 \frac{1}{2}$ facher Vergröfserung.) 


\section{$2 \mathrm{BHL}$ Biodiversity Heritage Library}

1922. "Neunter Beitrag zur Coleopteren-Fauna der Philippinen." Deutsche entomologische Zeitschrift 1922, 36-45.

https://doi.org/10.1002/mmnd.192219220104.

View This Item Online: https://www.biodiversitylibrary.org/item/103380

DOI: https://doi.org/10.1002/mmnd.192219220104

Permalink: https://www.biodiversitylibrary.org/partpdf/41396

\section{Holding Institution}

Harvard University, Museum of Comparative Zoology, Ernst Mayr Library

\section{Sponsored by}

Biodiversity Heritage Library

\section{Copyright \& Reuse}

Copyright Status: Public domain. The BHL considers that this work is no longer under copyright protection.

This document was created from content at the Biodiversity Heritage Library, the world's largest open access digital library for biodiversity literature and archives. Visit BHL at https://www.biodiversitylibrary.org. 